(C) Springer (India) Pvt. Ltd. 2018

Vishali Gupta

Quan Dong Nguyen

'Phuc LeHoang

and

Carl P Herbort Jr.

The Uveitis Atlas

$10.1007 / 978-81-322-2506-5 \quad 105-1$

\title{
Bilateral Diffuse Uveal Melanocytic Proliferation (BDUMP)
}

Rohan Bir Singh $^{1}$, Aniruddha Agarwal ${ }^{2}$, Rupesh Agrawal ${ }^{3,4}$, Mandeep Sagoo ${ }^{4,5}$, Kanika Aggarwal $^{2}$ and Vishali Gupta ${ }^{2}$

(1)Schepens Eye Research Institute, Massachusetts Eye and Ear Infirmary, Department of Ophthalmology, Harvard Medical School, Boston, MA, USA

(2)Advanced Eye Center, Post Graduate Institute of Medical Education and Research (PGIMER), Chandigarh, India

(3)National Healthcare Group Eye Institute, Tan Tock Seng Hospital, Singapore, Singapore

(4)Department of Medical Retina, Moorfields Eye Hospital NHS Foundation Trust, London, UK

(5)UCL Institute of Ophthalmology, London, UK

\section{Rohan Bir Singh}

Email: dr.rohandhaliwal@gmail.com

Aniruddha Agarwal

Email: aniruddha9@gmail.com

Rupesh Agrawal

Email: rupeshttsh@gmail.com

Mandeep Sagoo

Email: Mandeep.Sagoo@moorfields.nhs.uk

Kanika Aggarwal

Email: kanika2k1@yahoo.co.in

Vishali Gupta (Corresponding author)

Email: vishalisara@yahoo.co.in

Email: vishalisara@gmail.com

\section{Abstract}

Bilateral diffuse uveal melanocytic proliferation (BDUMP) is a rare paraneoplastic syndrome characterized by multiple subretinal, round, red patches in the retinal pigment epithelium; hyperfluorescence on fluorescein angiography; multiple elevated pigmented and nonpigmented uveal 
melanocytic tumors with diffuse uveal tract thickening; exudative retinal detachments; and rapid cataract development leading to progressive loss of vision. BDUMP is predictive of an occult or undiagnosed systemic malignancies and a marker for poor prognosis. The index chapter describes an illustrative case of BDUMP with multimodal imaging.

Keywords Paraneoplastic syndrome - Bilateral diffuse uveal melanocytic proliferation Melanocytic tumors - Uveal tumors - BDUMP - Melanocytes - Melanoma - Uvea

\section{Introduction}

Bilateral diffuse uveal melanocytic proliferation (BDUMP) is a rare paraneoplastic ocular syndrome that may lead to profound vision loss and benign proliferation of choroidal melanocytes. The proliferated melanocytes are histopathologically unrelated to the nonocular, systemic tumors. Typically, the five characteristic clinical signs of the disease are multiple subretinal, round, red patches in the retinal pigment epithelium (RPE); early fluorescence of these lesions on fluorescein angiography; multiple elevated pigmented and nonpigmented uveal melanocytic tumors with diffuse uveal tract thickening; exudative retinal detachments; and rapid cataract development.

Most of the reported cases of BDUMP present as bilateral vision loss in adult patients beyond the 5th decade. In majority of these cases, pigmented choroidal lesions are observed. Initial vision loss is followed by bilateral exudative retinal detachment and rapid progression of the cataract. The management involves surgical removal of cataracts, and resolution of associated retinal detachment with treatment of associated systemic malignancy may restore the vision to a limited degree. However, BDUMP is often associated with occult but advanced carcinoma, an indicator of poor prognosis. The ocular manifestations precede systemic carcinoma by 3-12 months, hence emphasis is laid on prompt systemic evaluation. The underlying cause of retinal detachment is not known, but is attributed to the malfunctioning retinal pigment epithelium (RPE) and breakdown of the blood-retina barrier. The development of rapid cataract, within 2-3 months, is considered to be caused from ciliary body involvement, leading to inadequate aqueous volume, poor nutrient composition, or release of toxins.

Case 1

A 62-year-old female, presented with a history of visual disturbances and reduction of vision in the left eye for the past 1 year. Best-corrected visual acuity (BCVA) was 6/6 in the right eye and 6/18 in the left eye. Mild posterior subcapsular cataract was noted in the left eye. The anterior segment was quiet in both eyes.

Deep, pigmented lesions were noted in the left eye on posterior segment evaluation. These lesions were at the level of the retinal pigment epithelium (RPE) with diffuse distribution in both eyes (Fig. 1). Fluorescein angiography (FA) showed the presence of multiple discrete and partially confluent hyperfluorescent lesions representing RPE window defects (Fig. 2). In the right eye these lesions were present in the macula. Indocyanine green angiography demonstrated early and late hypofluorescent spots corresponding with multiple pigmented lesions (Fig. $\underline{3}$ ). Optical coherence tomography (OCT) demonstrated presence of significant subfoveal fluid in the left eye as well as RPE disruptions in both the eyes (Fig. 4). The RPE disruptions were co-localized to the pigmented plaques on the fundus and the areas of window defects on fluorescein angiography. Fundus autofluorescence showed hypoautofluorescence in these regions. 

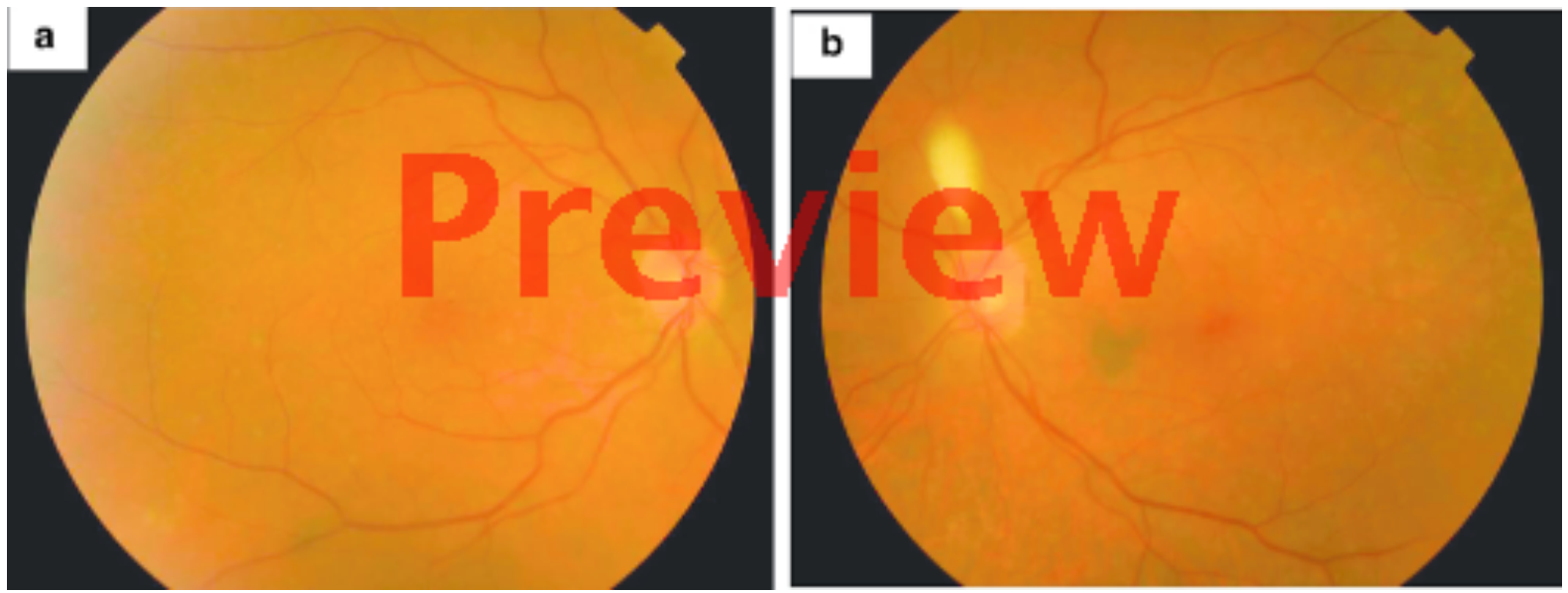

If you need to edit the image, please use the original: 340020_0_En_105-1_Fig1_Print.tif

Fig. 1

Color fundus photographs of both eyes of the patient shows presence of multiple, round, deep, pigmented lesions at the level of the retinal pigment epithelium. The lesions are faint grey to brown in appearance and large in size

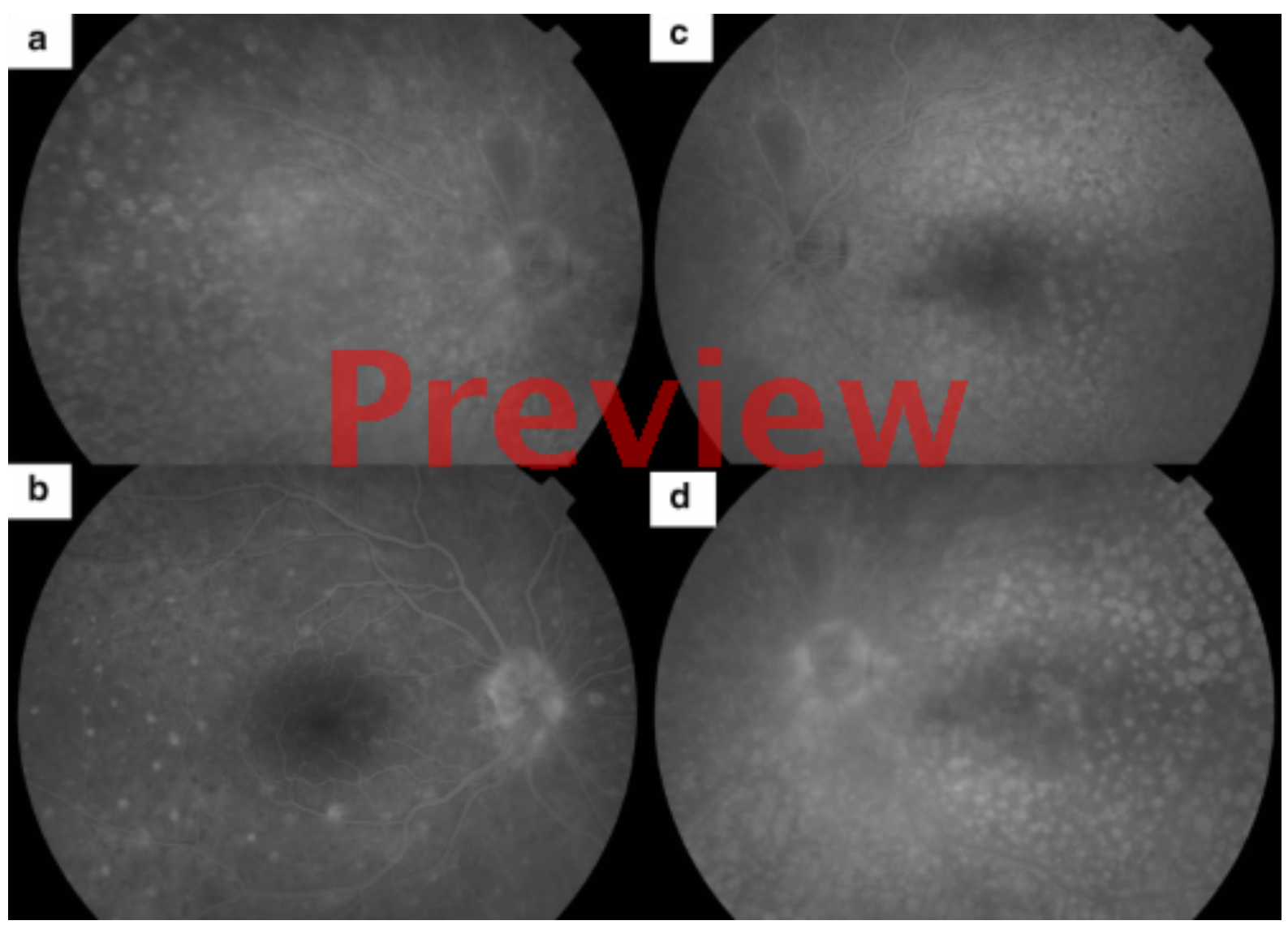

If you need to edit the image, please use the original: 340020_0_En_105-1_Fig2_Print.tif

Fig. 2

Fluorescein angiography of both the eyes shows presence of hyperfluorescence in the area of pigmented lesions that remain hyperfluorescent in the late phase, suggestive of retinal pigment epithelium window defects. Right eye is shown in (a) and (b), and left eye in (c) and (d) 


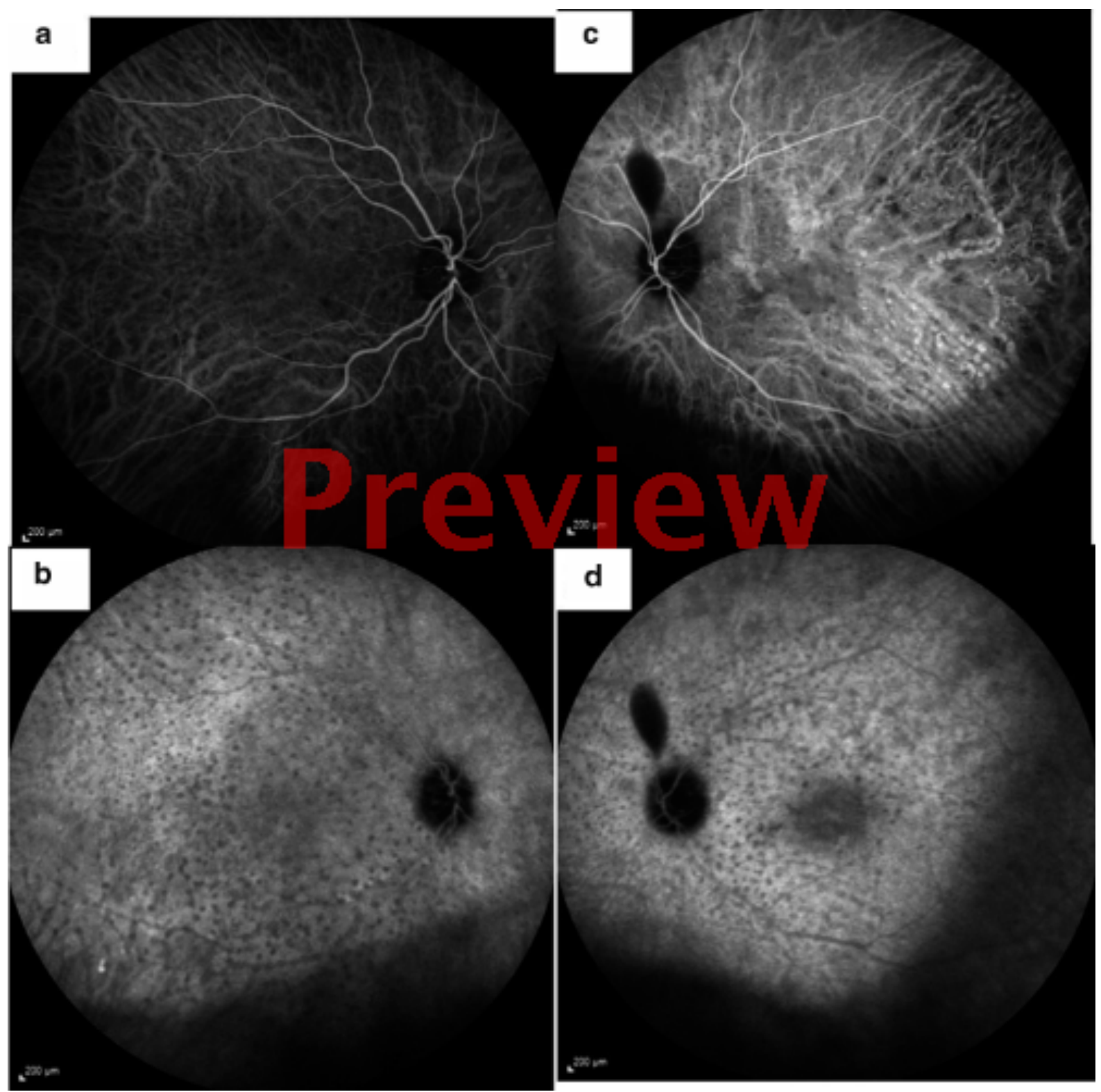

If you need to edit the image, please use the original: 340020_0_En_105-1_Fig3_Print.tif

Fig. 3

Indocyanine green angiography shows presence of numerous hypocyanescent lesions in the early and late phase corresponding to the retinal pigment epithelium (RPE) window defects ( $a$ and $b$ ). Fundus autofluorescence imaging shows more numerous hypo-autofluorescent lesions due to the atrophy of the RPE (c and d) 


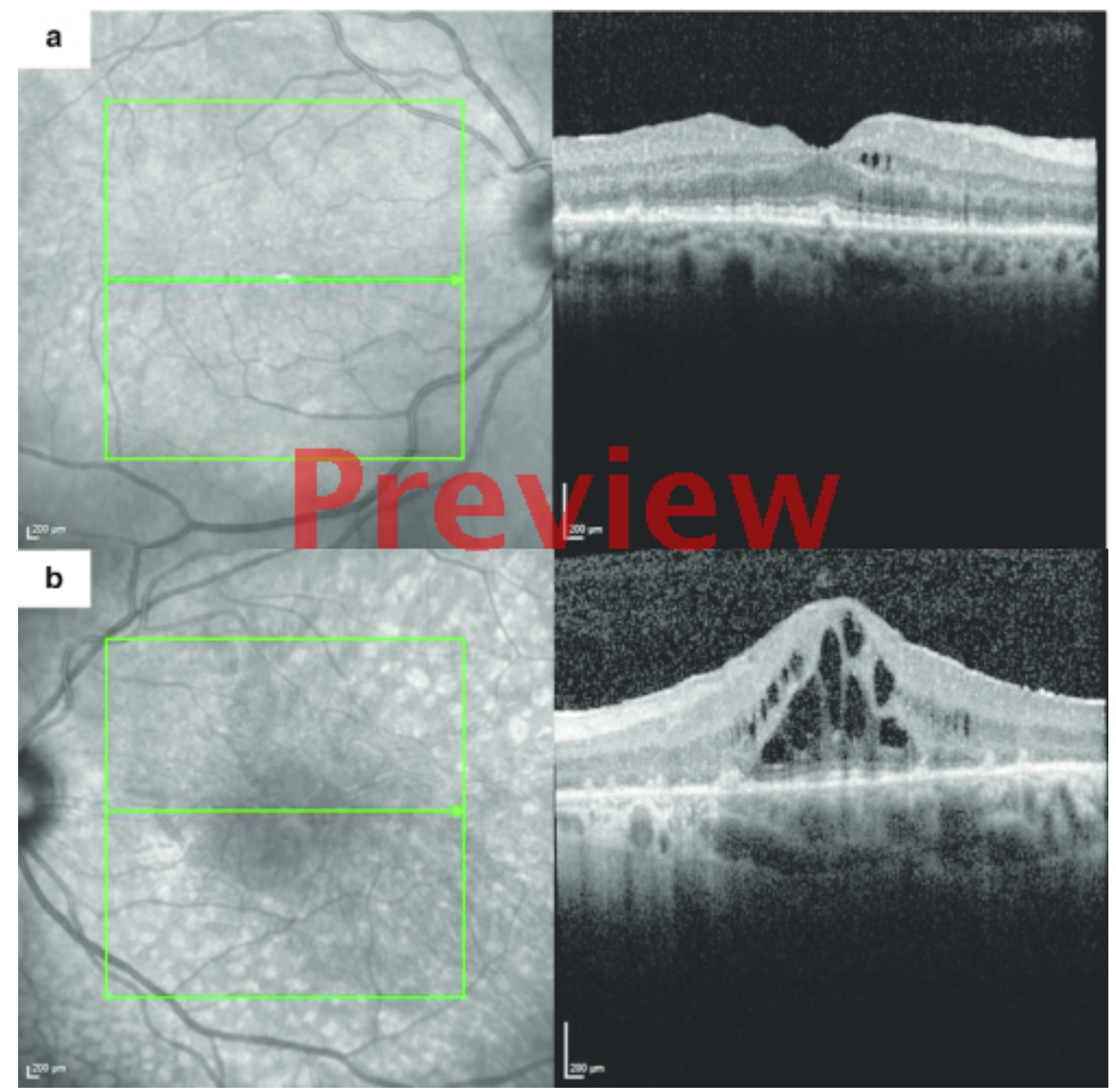

If you need to edit the image, please use the original: 340020_0_En_105-1_Fig4_Print.tif

Fig. 4

Spectral-domain optical coherence tomography (OCT) scans of the right (a) and left (b) eyes shows presence of retinal pigment epithelial (RPE) disturbances with excrescences at the level of the RPE and presence of subretinal fluid in the left eye

The differential diagnosis included metastasis. White dot syndromes were ruled out due to absence of any activity of the lesions. The differential diagnosis of bilateral intraocular lymphoma in both eyes was also considered. Systemic evaluation revealed presence of cerebellar symptoms. MRI of the brain showed presence of brainstem and right cerebellopontine lesions suggestive of central nervous system lymphoma. Biopsy from the brain revealed large B-cells positive for CD20, CD3 negative, CD79a positive, CD10 negative, bcl-6 positive, MUM1 positive, bcl-2 positive, and EBER negative. The histopathological findings were consistent with diffuse large B-cell lymphoma (DLBCL) of the brain. The patient was referred to Hemato-oncology and started on chemotherapy.

While there was improvement in macular edema, the fundus lesions did not change following initiation of chemotherapy, which was tolerated well by the patient.

(Case report was published in: Pefkianaki et al. ( 2015)) 
Key Points

1.

Bilateral diffuse uveal melanocytic proliferation (BDUMP) is a rare, paraneoplastic syndrome affecting the posterior segment resulting in progressive visual loss.

2.

BDUMP is associated with characteristic, multiple subretinal, round, red patches in the retinal pigment epithelium (RPE); elevated pigmented and nonpigmented uveal melanocytic tumors with diffuse uveal tract thickening; and exudative retinal detachments.

3.

BDUMP is often associated with occult but advanced carcinoma, and is an indicator of poor prognosis.

\section{Suggested Reading}

Klemp K, Kiilgaard JF, Heegaard S, Nørgaard T, Andersen MK, Prause JU. Bilateral diffuse uveal melanocytic proliferation: case report and literature review. Acta Ophthalmol. 2017;95(5):439-45. CrossRef PubMed

Mittal R, Cherepanoff S, Thornton S, Kalirai H, Damato B, Coupland SE. Bilateral diffuse uveal melanocytic proliferation: molecular genetic analysis of a case and review of the literature. Ocul Oncol Pathol. 2015;2(2):94-9.

CrossRef PubMed PubMedCentral

Pefkianaki M, Agrawal R, Desai P, Pavesio C, Sagoo MS. Bilateral diffuse uveal melanocytic proliferation (BDUMP) associated with B-cell lymphoma: report of a rare case. BMC Cancer. 2015;15:23.

CrossRef PubMed PubMedCentral

Saito W, Kase S, Yoshida K, Ohguro H, Yokoi M, Iwaki H, Ohno S. Bilateral diffuse uveal melanocytic proliferation in a patient with cancer-associated retinopathy. Am J Ophthalmol. 2005;140(5):942-5.

CrossRef PubMed 